\title{
Resultados al Tratamiento de Linfoma de Burkitt en Pacientes Pediátricos del Hospital Escuela Universitario Durante el Periodo Enero 2005 - Diciembre 2016.
}

Results to the Treatment of Burkitt's Lymphoma in Pediatric Patients of the University School Hospital during the period January 2005- December 2016.

\author{
Videlda Grissel Aguilar Reyes* (D) , Dorcas Judit Arguijo Armijo* (D) \\ * Pediatra, Hospital Enrique Aguilar Cerrato, Esperanza, Intibuca, Honduras. \\ * Pediatra, Por Salud, Hospital Maria, Especialidades Pediátricas.
}

\section{RESUMEN}

Introducción.- El Linfoma de Burkitt (LB) es un Linfoma no Hodgkin altamente agresivo y es el tumor humano de más rápido crecimiento. Su forma de presentación más habitual es la enfermedad ganglionar. El tratamiento es uno de los grandes éxitos de la oncología, siguiendo protocolos liderados por sociedades de oncología pediátrica.

Objetivos.- Determinar las Características Clínicas y Epidemiológicas de pacientes pediátricos con Linfoma de Burkitt tratados en el Hospital Escuela Universitario-Bloque Materno Infantil en el periodo Enero 2005-Diciembre 2016. Metodología. Estudio descriptivo, transversal, retrospectivo, en paciente pediátricos con Linfoma de Burkitt, tratados con el protocolo AHOPCA en el servicio de Hematooncología del Hospital Materno Infantil, muestreo no probabilístico por conveniencia. Protocolo revisado por el Comité de Bioética/ Instituto Hondureño de Seguridad Social. Los datos recolectados se ingresaron en paquete estadístico EPI-INFO.

Resultados.- Las edades encontradas fueron desde los 3 a 17 años de edad, 63.4\% fueron hombres, con una relación masculino/Femenino de 1.9:1. La manifestación clínica más frecuente fueron masas palpables $87.8 \%, 19.5 \%$ se encontraron en estadio I, el $14.6 \%$ tuvieron diagnóstico de estadio II, estadio III fueron el $51.3 \%$, estadio IV lo encontramos en un $14.6 \%$ Remisión completa de proceso patológico del $85.4 \%$, no tuvimos recaídas ni fallas tumorales iniciales.

Conclusiones.- A pesar de tratarse de un grupo de enfermedades de alto grado de malignidad, con alta proliferación celular y por tanto de creci-

\footnotetext{
Detalle de los Autores:

Videlda Grissel Aguilar Reyes, Dorcas Judit Arguijo Armijo

MD. Residente del Tercer año Postgrado de Pediatría. Facultad de Ciencias

Médicas. Universidad Nacional Autónoma de Honduras. 2017-2019.

Correspondencia:

Videlda Grissel Aguilar Reyes, Hospital Enrique Aguilar Cerrato, La Esperanza,

Intibuca, Honduras. / grissel2488@gmail.com
}

miento acelerado, la respuesta al tratamiento de esta patología suele ser excelente logrando una sobrevivencia alta a pesar de un país subdesarrollado.

Palabras Claves.- Honduras, Linfoma de Burkitt, pediatría.

\section{ABSTRACT}

Introduction.- Burkitt lymphoma is a highly aggressive non-Hodgkin's lymphoma and is the fastest growing human tumor. Its most common form of presentation is ganglion disease. Treatment is one of the great successes of oncology, following protocols led by societies of pediatric oncology.

Objective.- To determine the clinical and epidemiological characteristics of pediatric patients with Burkitt's lymphoma treated at the Hospital Escuela Universitario - Bloque Materno Infantil Hospital during the period January 2005December 2016.

Methodology:- Descriptive study in pediatric patients with Burkitt's lymphoma, treated with the AHOPCA 2005 protocol in the Hemaoncology service of the Maternal and Child Hospital, non-probabilistic sampling for convenience. protocol reviewed by the Bioethics Committee / IHSS. The data collected were entered in the EPI-INFO statistical package.

Results.- The ages found were from 3 to 17 years old, $63.4 \%$ were men, with a male / female ratio of 1.9: 1 . The most frequent clinical manifestations were palpable masses $87.8 \%$, $19.5 \%$ were in stage I, $14.6 \%$ had a diagnosis of stage II, stage III were $51.3 \%$, stage IV was found in $14.6 \%$ Complete remission of pathological process of $85.4 \%$, we had no relapses or initial tumor failures.

Conclusions.- Despite being a group of diseases with a high degree of malignancy, with high cell proliferation and therefore accelerated growth, the response to treatments is usually 
excellent due to the timely management of this condition in our healthcare center; achieving a high survival despite an underdeveloped country.

Key Words.- Honduras, Burkitt's lymphoma, pediatrics

\section{Introducción}

El cáncer infantil ocupa la segunda causa de mortalidad en niños menores de 15 años. La leucemia es el cáncer más común, seguido de los linfomas y tumores cerebrales que ocupan el tercer grupo de neoplasias por incidencia en la edad infantil. Los linfomas se clasifican en linfoma Hodgkin y linfoma no Hodgkin. Los linfomas no Hodgkin (LNH) representan el 7\%, entre ellos se encuentran el linfoma Burkitt, el linfoma difuso de células grandes y el linfoma linfoblástico. El linfoma Burkitt es el tercer tumor de origen linfoide. Representa entre el 40 a $50 \%$ de los linfomas no Hodgkin y el $3-4 \%$ de todas las neoplasias infantiles diagnosticadas cada año en los Estados Unidos de América. En los niños africanos, la incidencia estimada es de 3 a 6 casos por cada 100.000 niños/año, esta patología fue descubierta por primera vez en niños y adolescentes africanos. Se describen 3 variantes según la OMS: La esporádica, la endémica que predomina en África asociada al virus de Epstein Barr y el Plasmodium falciparum y la variante asociada a inmunodeficiencia, se observa principalmente en pacientes infectados con Virus de Inmunodeficiencia Adquirida $(\mathrm{VIH})$. Aunque estas formas presentan características clínicas diferentes, tienen en común la elevada frecuencia de afectación extra nodal. EI LB esporádico se presenta, en la mayoría de los casos, como una masa abdominal simulando la forma de presentación de la apendicitis aguda o invaginación intestinal, también afecta con frecuencia la región para faríngea y paranasal. A diferencia con la forma endémica, la afectación mandibular no es frecuente. Otros lugares menos habituales de presentación son; la piel, el SNC y el hueso.

El tratamiento de los $\mathrm{LNH}$ es uno de los grandes éxitos de la oncología. Actualmente la supervivencia a nivel mundial en linfoma de Burkitt es entre $90-95 \%$ de los casos diagnosticados en estadios localizados y en el $70-90 \%$ en los casos diseminados. En nuestro medio no existen estudios descriptivos ni analíticos que valoren la eficacia, ni la incidencia de los efectos adversos en los diferentes esquemas de tratamiento empleado en el linfoma de Burkitt por lo que nos planteamos la interrogante ¿Cuáles son los resultados al tratamiento de los pacientes pediátricos con linfoma de Burkitt tratados en el Hospital Escuela Universitario en el periodo Enero 2005 - Diciembre 2016?

Para estadificarlo se utiliza la escala de estatificación de Murphy:

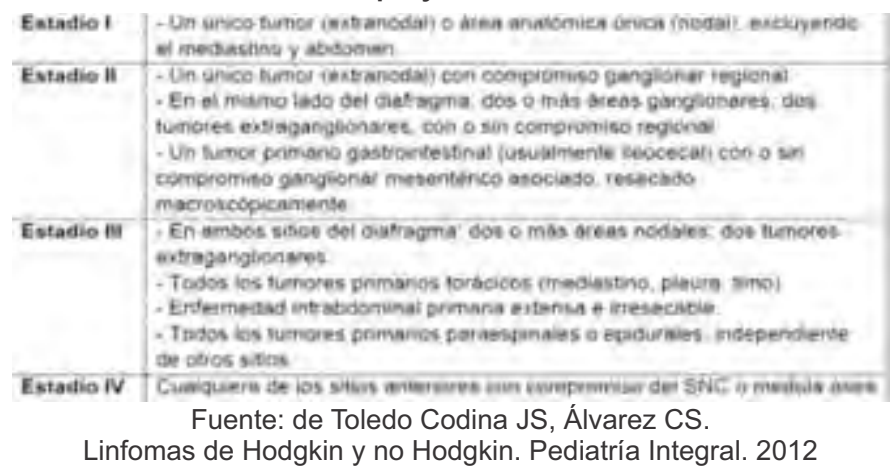

\section{Metodologia}

Se trata de un estudio descriptivo, transversal, retrospectivo, desde enero del año 2005 a diciembre 2016. Fueron tomados en cuenta 41 expedientes clínicos, que contaban con la información mínima necesaria para formar parte del estudio, de acuerdo a los criterios de inclusión, mediante un muestro no Probabilístico por conveniencia. Las variables estudiadas fueron datos generales y socio-demográficos, antecedentes de cáncer familiar, fecha de diagnóstico, presencia de Inmunodeficiencia, antecedentes neonatales e inmunológicos, así mismo determinar los síntomas/signos constitucionales, sitio anatómico de compromiso tumoral nodal y extra nodal, estadio, infiltración de sitios anató-micos, valor de LDH, tratamiento de protocolo establecido de acuerdo al estadio, con la revisión de las fechas de inicio como de finalización, si se cumplió los ciclos en el tiempo correspondiente, respuesta al tratamiento, si se tuvo remisión completa o parcial, recaída, muerte en inducción o si se encuentra en vigilancia. Los criterios de inclusión que fueron tomados en cuenta fueron: Todos los pacientes menores de 18 años de edad, con diagnostico histopatológico de Linfoma de Burkitt en el Hospital Escuela Universitario tratados con el protocolo del grupo AHOPCA año 2005, en el servicio de Hematooncología en el tiempo establecido. La tabulación de los datos fue 
a través del programa Excel $\circledast$ para Windows 10 Home, versión 1809; el análisis de datos usamos el programa estadístico EPI-INFO $®$ versión 7.2.2.16. El protocolo de investigación fue presentado al Comité de Bioética y Gerencia de Docencia e Investigación del Instituto Hondureño de Seguridad Social.

\section{Resultados}

Las edades de los pacientes estudiados estuvieron comprendidas desde los 3 años hasta los 17 años de edad. El rango de edad más frecuente fue entre los 5 a 9 años, con una media aritmética de 8.10 años, mediana de 6 , moda de 4 años y desviación estándar del 4.54, en un intervalo de confianza del $95 \%$, el rango de edad de menos frecuencia fueron los mayores de 15 años (5 casos nada más). En la distribución por sexo, se obtuvo $63.4 \%$ fueron hombres, con una relación masculino/Femenino de 1.9:1. Hablando del grado o nivel socioe-conómico, nos encontramos con un $97.6 \%$ están en el grado de pobreza, predomina el área urbana (52.1.\%), 5 casos $(12.2 \%)$ presentaron antecedentes familiares de cáncer.

En cuanto al estadio clínico al momento de hacer el diagnostico se encontró que 21 pacientes $(51.3 \%)$ estaban en el estadio III de la enfermedad. Las manifestaciones clínicas más frecuentes fueron; la presencia de masas palpables en 36 casos $(87.8 \%)$, fiebre en 17 pacientes $(41.5 \%)$ y adenopatías en 12 casos (29.3\%), en menor frecuencia pérdida de peso en 7 pacientes los cuales 5 pacientes estaban en estadio III y ascitis 2 casos (4.9\%). Ver Cuadro No 1.

Cuadro No 1. Estadio y Manifestaciones Clínicas Constitucionales más frecuentes en pacientes con diagnóstico y tratamiento de Linfoma de Burkitt, Servicio de Hematooncología Pediátrica, Hospital Escuela Universitario-Bloque Materno Infantil. 20052016. $\mathrm{N}=41$.

\begin{tabular}{|c|c|c|c|c|c|c|}
\hline \multicolumn{7}{|c|}{ Manifestaciones Clínicas } \\
\hline Estadio & Fiebre & Pérdida de Peso & Respiratoria & Ascitis & Adenopatias & Masas \\
\hline & $\mathrm{N}(\%)$ & $\mathrm{N}(\%)$ & $\mathrm{N}(\%)$ & $\mathrm{N}(\%)$ & $\mathrm{N}(\%)$ & $\mathrm{N}(\%)$ \\
\hline Estadio I & $1(2.4)$ & $1(2.4)$ & $1(2.4)$ & $0(0.0)$ & $2(4.9)$ & $7(17.1)$ \\
\hline Estadio II & $4(9.8)$ & $0(0.0)$ & $0(0.0)$ & $0(0.0)$ & $4(9.8)$ & $6(14.6)$ \\
\hline Estadio III & $10(24.4)$ & $5(12.3)$ & $0(0.0)$ & $2(4.9)$ & $3(7.3)$ & $17(41 \% .5)$ \\
\hline Estadio IV & $2(4.9)$ & $1(2.4)$ & $2(4.9)$ & $0(0.0)$ & $3(7.3)$ & $6(14.6)$ \\
\hline
\end{tabular}

La estadificacion de linfoma de Burkitt se realiza de acuerdo a sitios tomados y afectación ganglionar, en nuestro estudio, encontramos que 8 casos (19.5\%) se encontraron en estadio I, 6 casos (14.6\%) tuvieron diagnóstico de estadio II, en estadio III 21 casos (51.3\%), el estadio IV encontramos 6 casos (14.6\%). En relación al compromiso tumoral al momento del diagnóstico se encontró que 30 pacientes $(73.4 \%)$ tenían compromiso extra nodal y 11 compromiso nodal (26.6\%). Cuadro Nº 2.

Cuadro No 2. Clasificación del estadio y afectación nodal y extranodal en paciente con diagnóstico y tratamiento de Linfoma de Burkitt, Servicio de Hematooncología Pediátrica, Hospital Escuela Universitario-Bloque Materno Infantil. 2005-2016. $\mathrm{N}=41$.

\begin{tabular}{ccc}
\hline & \multicolumn{2}{c}{ Compromiso Tumoral } \\
\cline { 2 - 3 } Estadio & Nodal & Extra nodal \\
& $\mathbf{N}(\%)$ & $\mathbf{N}(\%)$ \\
\hline Estadio I & $4(9.8)$ & $4(9.8)$ \\
Estadio II & $2(4.9)$ & $4(9.8)$ \\
Estadio III & $3(7.3)$ & $18(44.0)$ \\
Estadio IV & $2(4.9)$ & $4(9.8)$ \\
\hline
\end{tabular}

Fuente: Encuesta realizada por los autores

En cuanto al cumplimiento de tratamiento en el tiempo establecido encontramos que 32 pacientes $(78.0 \%)$ no tuvieron retraso en los ciclos de tratamiento, de estos el $48.8 \%$ fueron niños y el $29.3 \%$ niñas. El resto de los pacientes tuvo retraso en los ciclos de tratamiento por diferentes causas, predominando la de origen infecciosa, siendo las principales: Bacteremia/mucositis, neumonía, bronquitis aguda, neutropenia febril e Insuficiencia renal aguda. Cuadro $N^{\circ} 3$.

Cuadro No 3. Causas del retraso del tratamiento en el tiempo establecido en paciente con diagnóstico de Linfoma de Burkitt, Servicio de Hematooncologia Pediátrica, Hospital Escuela Universitario-Bloque Materno Infantil. 2005-2016. N=41. 


\begin{tabular}{|ccccccc|}
\hline & \multicolumn{2}{c}{$\begin{array}{c}\text { Tratamiento } \\
\text { Cumplido }\end{array}$} & \multicolumn{2}{c|}{ Causa del No cumplimiento del tratamiento } \\
\hline Sexo & $\mathrm{Sl}$ & $\mathrm{N} 0$ & Bac $^{*}+\mathrm{Muc}^{*}$ & Broq $^{q}+\mathrm{Neu}^{\beta}$ & $\mathrm{N}^{\mathrm{f}}+\mathrm{Neu}+\mathrm{R} \mathrm{R}^{\varepsilon}$ & $\mathrm{Neu}$ \\
& $\mathrm{N}(\%)$ & $\mathrm{N}(\%)$ & $\mathrm{N}(\%)$ & $\mathrm{N}(\%)$ & $\mathrm{N}(\%)$ & $\mathrm{N}(\%)$ \\
\hline \multirow{2}{*}{ Masculino } & 20 & 6 & 0 & 1 & 0 & 4 \\
& $(48.8)$ & $(14.6)$ & $(0.0)$ & $(2.4)$ & $(0.0)$ & $(9.8)$ \\
\hline \multirow{2}{*}{ Femenino } & 12 & 3 & 1 & 0 & 1 & 1 \\
& $(29.3)$ & $(7.3)$ & $(2.4)$ & $(0.0)$ & $(2.4)$ & $(2.4)$ \\
& & & & & & \\
\hline
\end{tabular}

Fuente: Encuesta por los autores

*Bac: Bacteremia ¥Muc: Mucositis aBronq: Bronquitis Aguda

BNeu: Neutropenia Febril $€ N$ : Neumonía £IRA: Insuficiencia Renal Aguda

De acuerdo a la respuesta al tratamiento con el protocolo establecido en relación al estadio clínico, encontramos una remisión completa de proceso patológico en 35 pacientes (85.4\%), así mismo se encuentran en estado de vigilancia 25 actualmente en el servicio de Hemato-oncología Pediátrica, no tuvimos recaídas, ni fallas tumorales en los pacientes que estuvieron en tratamiento durante el tiempo estudiado ni en el seguimiento. Cuadro $\mathrm{N}^{\circ} 4$.

Cuadro No 4. Remisión, falla tumoral y recaídas en paciente con diagnóstico y tratamiento de Linfoma de Burkitt, Servicio de Hematooncología Pediátrica, Hospital Escuela Universitario-Bloque Materno Infantil. 20052016. $N=41$.

\begin{tabular}{|cccccccccc|}
\hline & $\begin{array}{c}\text { Remisión } \\
\text { Completa }\end{array}$ & \multicolumn{2}{c}{$\begin{array}{c}\text { Remisión } \\
\text { Parcial }\end{array}$} & \multicolumn{2}{c}{$\begin{array}{c}\text { Falla } \\
\text { tumoral }\end{array}$} & \multicolumn{2}{c|}{ Recaídas } \\
\hline Sexo & $\mathrm{SI}$ & $\mathrm{NA}$ & $\mathrm{NO}$ & $\mathrm{NA}$ & $\mathrm{NO}$ & $\mathrm{NA}$ & $\mathrm{N} 0$ & $\mathrm{NA}$ \\
& $\mathrm{N}(\%)$ & $\mathrm{N}(\%)$ & $\mathrm{N}(\%)$ & $\mathrm{N}(\%)$ & $\mathrm{N}(\%)$ & $\mathrm{N}(\%)$ & $\mathrm{N}(\%)$ & $\mathrm{N}(\%)$ \\
\hline \multirow{2}{*}{ Masculino } & 23 & 3 & 22 & 4 & 22 & 12 & 22 & 12 \\
& $(56.1)$ & $(7.3)$ & $(53.7)$ & $(9.8)$ & $(53.7)$ & $(29.3)$ & $(53.7)$ & $(29.3)$ \\
\hline \multirow{2}{*}{ Femenino } & 12 & 3 & 12 & 3 & 4 & 3 & 4 & 3 \\
& $(29.3)$ & $(7.3)$ & $(29.3)$ & $(7.3)$ & $(9.8)$ & $(7.3)$ & $(9.8)$ & $(7.3)$ \\
\hline
\end{tabular}

Fuente: Encuesta por los autores

${ }^{*}$ N/A: No Aplica

Remisión completa: Desaparición de todos los signos del cáncer en respuesta al tratamiento

Remisión parcial: Disminución del tamaño de un tumor o en el grado del cáncer en el cuerpo, en respuesta al tratamiento

Recaída: Reaparición de una enfermedad o de los signos y síntomas de una enfermedad después de un período de mejoría

Al hablar del estado actual de los pacientes, observamos que 25 pacientes $(61.0 \%)$ se encuentran todavía en estado activo por el servicio de Hematooncología Pediátrica, ya que periódi- camente son evaluados en consulta externa para su seguimiento. 6 pacientes $(14.6 \%)$ ya fueron dados de alta completamente, 3 pacientes falle-cieron (7.3\%) procedentes de los departamentos de Comayagua, Choluteca y Francisco Morazán; el primer caso se catalogó como estadio I, segundo caso estadio III, tercer caso estadio IV. uno de ellos tuvo bronquitis aguda neutropenia febril y el otro neumonía, neutropenia febril más lesión renal aguda. Cuadro $N^{\circ} 5$.

Cuadro No 5. Estado actual de los pacientes diagnosticados con Linfoma de Burkitt, Servicio de Hemato-oncología Pediátrica, Hospital Escuela Universitario-Bloque Materno Infantil. 2005-2016. $\mathrm{N}=41$.

\begin{tabular}{ccccc}
\hline & \multicolumn{4}{c}{ Estado Actual del paciente } \\
\cline { 2 - 5 } Estadio & Activo & Alta & Muerte & Perdida \\
& $\mathbf{N}(\%)$ & $\mathbf{N}(\%)$ & $\mathbf{N}(\%)$ & $\mathbf{N}(\%)$ \\
\cline { 2 - 5 } Estadio I & $6(14.6)$ & $0(0.0)$ & $1(2.4)$ & $1(2.4)$ \\
Estadio II & $5(12.3)$ & $0(0.0)$ & $0(0.0)$ & $1(2.4)$ \\
Estadio III & $12(29.3)$ & $5(12.3)$ & $1(2.4)$ & $2(4.9)$ \\
Estadio IV & $2(4.9)$ & $1(2.4)$ & $1(2.4)$ & $2(4.9)$ \\
\hline
\end{tabular}

Fuente: Encuesta realizada por los autores

En relación a la procedencia y estado actual del paciente encontramos que 14 pacientes (34.1\%) son procedentes de Francisco Morazán, (10 en estado activo de su tratamiento, 3 perdidos y un muerto). 5 pacientes (12.2\%) procedentes de Choluteca, de estos 3 activos una perdida y 1 muerto. Tuvimos 5 pacientes (12.2\%) procedente de Comayagua (2 altas y 1 activo, 1 perdido y 1 muerto).

\section{Discusion}

En esta investigación se estableció conocer sobre el Linfoma de Burkitt, describir sus factores de riesgo, complicaciones más frecuentes, así también conocer las manifestaciones clínicas más comunes y la respuesta al tratamiento en pacientes del Servicio de Hematooncológica Pediátrica del Hospital Escuela Universitario - Bloque Materno Infantil, durante los años 2005-2016. Los primeros 37 casos reportados por linfoma de Burkitt en Honduras fueron realizados en el año 1985, estos fueron diagnosticados en el Hospital Escuela, en un lapso de 6 años. 
López et al, encontraron (14) que no presentaron linfadenopatia periférica, sin esplenomegalia y solo un caso presento hepatomegalia. Tres tenían invasión a medula ósea y uno afectación del siste-ma nervioso central, correspondiente también a lo encontramos en nuestra serie de casos.

El predominio del género masculino fue mayor en concordancia con la literatura; encontrando una relación de 1.9:1 entre niños/niñas. Las causas de esta heterogeneidad no son conocidas, sin embargo esta distribución de géneros en el cáncer infantil es un hecho que se conoce en muchos países en vías de desarrollo, particularmente entre los Linfoma No Hodking. Los resultados publicados por Bravo LE y cols, en su serie de más de 30 años, muestran que la incidencia de LB entre niños de 5-9 años fue casi tres veces mayor que en niñas, en nuestra serie la mayor diferencia se encontró entre el rango de 2-5 años con una relación 3:1.(15)

Otro aspecto interesante es la edad en la cual este linfoma se presenta, observando que la población afectada en los países en vía de desarrollo es de menor edad que los pacientes de países europeos y Estados Unidos. Una compilación de estudios comparando la edad media de presentación entre diferentes regiones mostró un estimado de 6,1 años en África contra 19,2 años en Estados Unidos, esto también se extrapola a nuestros datos, donde encontramos que el rango de edad más frecuente en nuestra población es entre 5 a 9 años años de edad, con una edad media de presentación de 8 años.

Para Klumb y cols (16), en su estudio conducido en el Instituto Nacional de Cáncer en Rio de Janeiro con una media de 6 años (1-16 años) y para Hassam y cols de 5 años (2-14 años). Estos hallazgos podrían sugerir que una edad temprana de presentación es una característica de los LB en Colombia y algunos países de Suramérica, probablemente por ciertos factores medioambientales entre estos una exposición temprana a la enfermedad del Virus del Epstein Barr.

Como era de esperarse en el estudio, el compromiso extra nodal es el más frecuente $(73.1 \%)$ entre los casos estudiados, de estos el sitio anatómico más frecuente encontrado fue en la cavidad abdominal en un $63.4 \%$ (24 casos), este hallazgo que es concordante otros estudios
(Araujo, 1996) (Pizza et al. 2008. 42 casos $(79.2 \%)$ (Cunha et al, 2012. 50 casos $(66,7 \%)$ ). El compromiso ganglionar más frecuente fue cervical en un $17 \%$ de nuestra población estudiada, esto también es concordante con otras series (47,8\%). (17) (18).

De acuerdo a la clasificación de St Jude/Murphy, el $51.2 \%$ de los pacientes fueron diagnosticados en estadios avanzando III. En una menor frecuencia, encontramos pacientes en estadio IV en un $14.6 \%$ (6 casos). Este diagnóstico en estadios avanzados parece ser una característica común en todos los casos de LB con un $66 \%$ en Estadios III-IV y $34 \%$ Estadios III, esto también nos lo reporta Swerdlow et al(19). Este diagnóstico en estadios avanzados parece ser una característica común en todos los casos de LB con un $70.2 \%$ en Estadios III-IV y $29.8 \%$ Estadios I-II.

Para regiones como Brasil el LB pediátrico es diagnosticado en estadio avanzado, con descripciones que van desde el 68,9\% (18) hasta el $90,5 \%$ (17), siendo los resultados de este estudio concordantes con la literatura. Aunque la edad de diagnóstico no mostró diferencias estadísticamente significativas entre los estadios, se observó una tendencia de menor edad entre estadios avanzados: esto concuerda a lo encontrado por Hassan y cols, con menor edad en estadios avanzados (III/IV) (media 4.5 vs. 7 años; Mann-Whitney test, $p=0.03$ ).

Se considera que todas las variables clínicas muestran un riesgo de infiltración a Sistema Nervioso Central y Medula Ósea (20) aunque algunos autores consideran que en el Linfoma de Burkitt endémico es rara la infiltración a Médula Ósea(21) y en el esporádico la infiltración a Sistema Nervioso Central(18). En este estudio la mayor proporción correspondió a casos con infiltración al Sistema Nervioso Central en un $14.6 \%$ seguido de médula $(12.2 \%)$.

El Linfoma de Burkitt es una neoplasia agresiva caracterizada por una significativa respuesta a la quimioterapia intensiva en niños y adolescentes. En esta serie el $85.3 \%$ se encontraron en remisión. El $7.3 \%$ (3 casos) fallecieron procedentes de los departamentos de Comayagua, Choluteca y Francisco Morazán; uno de ellos tuvo bronquitis aguda neutropenia febril y el otro neumonía, neutropenia febril más lesión renal aguda, dos de ellos se catalogó en 
estadio IV con infiltración al sistema nervioso central a la medula ósea, otro en estadio III.

Nuestros casos que tuvieron desenlace desfavorable (3 casos), fueron durante la inducción, Molyneux et al (21), encontraron que las muertes tuvieron recaída y ocurrieron en el primer año, esto es concordante con la literatura en donde el riesgo de recaída luego del primer año es menor del $5 \%$.

Por lo tanto creemos que la evaluación de sobrevida libre de evento en el primer año, es probablemente un buen indicador de sobrevida a largo plazo. Algunas de las características que han sido asociadas con un resultado adverso en niños fueron evidenciadas de forma significativa en este grupo de pacientes, como una edad mayor, estadio avanzado e infiltración del Sistema Nervioso Central $(p=0.005)$ o Médula Ósea $(p=0.008)$.

En este estudio, los hallazgos son cercanos a las observaciones en países en vía de desarrollo, por ejemplo en África la remisión fue del $90 \%$ y en Brasil del 92\% (22) entre población pediátrica. En los casos analizados la mortalidad fue relacionada con la inducción al tratamiento seleccionado de acuerdo al estadio clínico e infiltración. Otros autores consideran como causas de muerte, recaída y/o falla del tratamiento en países industrializados, las terapias incompletas y menos agresivas, además de la malnutrición la cual probablemente incrementa el riesgo de toxicidad e infecciones relacionada a la terapia(18).

En nuestro estudio encontramos que no se cumplieron algunos tratamiento de acuerdo al tiempo programa porque estos pacientes presentaron cuadros infecciosos. En este estudio no se evaluaron factores como el estado nutricional, sin embargo es una consideración para futuras investigaciones.

La búsqueda de antecedentes fue enfocada en especial a la identificación de casos asociados con inmunodeficiencias, en esta serie de 12 años encontramos que el $97.2 \%$ no presente este problema. Hablando acerca del cáncer en la familia encontramos solamente un $12.2 \%$ de casos tenía historia familiar mas no sabemos si eran familiares de primer y/o segundo grado. Al respecto, el papel de los antecedentes familiares de cáncer en la etiología de los tumores malignos hematopoyéticos en la infancia ha sido ampliamente investigado por Rudan y cols (15) quienes utilizando los datos del estudio ESCALE en Francia, concluyeron que una historia familiar de cáncer se asoció con un mayor riesgo de $\mathrm{LH}(\mathrm{OR}=1,5[1,0-2,2])$ y la $\mathrm{LNH}(\mathrm{OR}=1,8$ [ 1.3 a 2.5 ] $)$, pero no con Leucemias Agudas ( OR = 1,0 [ 0,9-1,2 ] ). Aunque el tipo y características de este estudio no permite dar conclusiones, podemos considerar que la historia familiar de cáncer en la población analizada fue un factor relevante y que estudios con otro diseño se requieren para confirmar una posible asociación en Linfoma de Burkitt pediátrico.

Por último, tenemos que saber que la progresión de la enfermedad en el linfoma no hodgkiniano no sigue un modelo predecible como sucede en la enfermedad de Hodgkin, por lo que desarrollar una estadificación con valor pronóstico ha sido un reto. La estadificación clínica propuesta por el equipo del hospital St. Jude ha sido ampliamente aceptada y no coincide con la estadificación Ann Arbor; así, un paciente en estadio IV de Ann Arbor puede ser un paciente en estadio III según St. Jude, porque el estadio IV según St. Jude se reserva para los niños con afectación del Sistema Nervioso Central o de la médula ósea, dado que cualquiera de estas dos afectaciones en el linfoma de Burkitt confiere un alto índice de recaídas comparado con otras áreas extranodales de la enfermedad. Por el contrario, los niños con una enfermedad avanzada, pero sin afectación de la médula ósea o del SNC (estadio III) tienen un excelente pronóstico con los tratamientos actuales(24).

\section{Conclusiones}

El linfoma de Burkitt en la población pediátrica analizada se caracteriza por afectar de forma predominante al género masculino, se presenta en su mayoría entre niños de 5 a 9 años de edad, con una edad media de 8 años. El compromiso más frecuente es la cavidad abdominal en región extra nodal. La localización nodal no se relaciona a inmunodeficiencias, antecedente no encontrado en ningún caso, pero es más frecuente a edades mayores. Esta enfermedad se diagnostica en estadios avanzados III-IV y cuando infiltra SNC o MO lo hace en su mayoría comprometiendo ambos compartimientos. 
Las muertes observadas durante la serie de casos en el tiempo de estudio, fue durante la inducción al tratamiento y estas muertes estaban seve-ramente comprometidos con infiltración especialmente al SNC y medula osea, en estados avanzados IV, y el otro en estadio III

Conflicto de Interés.- Los autores declaramos no tener conflictos de interés en relación a este artículo.

Contribuciones.- VGAR, DJAA se encargaron de la edición del protocolo así mismo, la recolección de datos, análisis e interpretación, edición del informe final así como sus conclusiones y recordaciones.

Agradecimientos.- Al Dr. Armando Peña y Dr. Manuel Sierra, asesor Temático y Metodológico por su inigualable apoyo y contribución a la realización de este estudio.

\section{Bibliografía}

1. Díaz VL, Castro-Delgado OE, Delgado MFC. Linfoma de Burkitt orbitario en una paciente de 3 años de edad. Pediatría. 2017;50(2).

2. Ibáñez MS, Asorey-García A, Bueso ES, Sánchez JG. Denis Parsons Burkitt: pasión por el saber. Arch Soc Esp Oftalmol. 2015;90(12):1089-1090.

3. Linfoma no Hodgkin en niños [Internet]. [citado 15 de mayo de 2019]. Disponible en: https://www.cancer.org/es/cancer/linfomano-hodgkin-en-ninos.html

4. Royero Arias MR. Características por imagen de tumores renales, en pacientes de la Fundación Hospital Pediátrico de la Misericordia. Estudio descriptivo de corte transversal [Tesis]. Universidad Nacional de Colombia-Sede Bogotá;

5. Medina-Ortega ÁP, López-Valencia D, Mosquera-Monje SL, Mora-Obando DL, Dueñas-Cuéllar RA. Virus de Epstein-Barr y su relación con el desarrollo del cáncer. latreia. 2017;30(2):131-145.

6. Miron I, Miron L, Lupu VV, Ignat A. Silent presentation of multiple metastasis Burkitt lymphoma in a child: A case report and review of the literature. Medicine (Baltimore). julio de 2017;96(28):e7518.

7. de Toledo Codina JS, Álvarez CS. Linfomas de Hodgkin y no Hodgkin. Pediatría Integral. 2012;
8. Saénz A, Andrade R, Torres M. Linfoma de Burkitt. Hipótesis Apunt Científicos Uniandinos. 2015;(19):18-21.

9. Arboine Ciphas M. Linfoma de burkitt: a propósito de un caso. Med Leg Costa Rica. 2017;34(1):325-231.

10. Caracterización de los síndromes linfoproliferativos B por citometría de flujo. Desafíos diagnósticos y nuevas estrategias de estudio. [Internet]. [citado 16 de mayo de 2019].

11. Leukemia \& Lymphoma Society [Internet]. [citado 16 de mayo de 2019]. Disponible en: https://www.lls.org/

12. Figure 4. Murphy/St. Jude's staging system. 32 [Internet]. ResearchGate. [citado 16 de mayo de 2019]. Disponible en: https://www.researchgate.net/figure/Murphy -St-Judes-staging-system-32_fig1_6135606

13. Verdecia Cañizarez, C. Satos Labarcerna, ME. Lam Diaz, RM. Comportamiento del linfoma no Hodgkin en la edad pediátrica [Internet]. [citado 16 de mayo de 2019].2015. Rev Cubana Pediatr;87(4).

14. Lopez DVC, Nolasco DRM, Figueroa M, Glazer D. El Linfoma de Burkitt en Honduras. 1985. 53.

15. Galvis Castro KT. Linfoma de Burkitt: características clínicas, patológicas y moleculares en población pediátrica [Thesis]. Universidad Nacional de ColombiaSede Bogotá;

16. Klumb CE, Hassan R, De Oliveira DE, De Resende LMM, Carriço MK, De Almeida Dobbin J, et al. Geographic variation in Epstein-Barr virus-associated Burkitt's lymphoma in children from Brazil. Int $\mathrm{J}$ Cancer. 2004;108(1):66-70.

17. Pizza M, Bruniera P, Luporini SM, Marcelino da Silva HR, Borsato ML, de Castro HC, et al. Detection of Epstein-Barr virus in children and adolescents with Burkitt's lymphoma by in situ hybridization using tissue microarrays. Hematology. 2008;13(2):114-118.

18. Cunha KCCMS, Oliveira MCLA, Gomes ACS, Castro LPF de, Viana MB. Clinical course and prognostic factors of children with Burkitt's lymphoma in a developing country: the experience of a single centre in Brazil. Rev Bras Hematol E Hemoter. 2012;34(5): 361-366. 


\section{ARTÍCULO ORIGINAL}

19. Swerdlow SH, Campo E, Pileri SA, Harris NL, Stein $\mathrm{H}$, Siebert $\mathrm{R}$, et al. The 2016 revision of the World Health Organization classification of Iymphoid neoplasms. Blood. 2016;127(20):2375-2390.

20Ferry JA. Burkitt's lymphoma: clinicopathologic features and differential diagnosis. The oncologist. 2006;11(4):375-383.

21. Molyneux EM, Rochford R, Griffin B, Newton $R$, Jackson $G$, Menon $G$, et al. Burkitt's I y $\mathrm{m} \mathrm{phom} \mathrm{a.} \mathrm{Th} \mathrm{e} \mathrm{L}$ a n c e t . 2012;379(9822):1234-1244.

22. Harif M, Barsaoui S, Benchekroun S, Bouhas $R$, Doumbé P, Khattab M, et al. Treatment of B-cell lymphoma with LMB modified protocols in Africa-report of the FrenchAfrican Pediatric Oncology Group (GFAOP). $P$ e d i a $t r$ B l o o d C a $n$ c e $r$. 2008;50(6):1138-1142.

23. Yürür-Kutlay $N$, Tuncalı $T$, Karabulut $H G$, Sadeghi F, Akalın I, Sağlam B, et al. Akut Miyeloid Lösemide Kromozomal Anomaliler: Tek Merkezden 417 Olgunun Sitogenetik Sonuçları.

24. Mata Fernandez, C. Linfoma de Burkitt: El tumor pediátrico más frecuente en África. Acta Pediatr Esp. 2008; 66(7): 322-326. 